\title{
Caracterización de la respuesta espectral de paneles planos de yoduro de cesio en radiología digital**
}

\section{Characterization of the spectral response of caesium iodide flat panel detectors in digital radiography}

\author{
Aitor Fernández Iglesias, Santiago Miquélez Alonso, Fernando Caudepón Moreno \\ Servicio de Radiofísica y Protección Radiológica. Hospital Universitario de Navarra - Nafarroako Unibertsitate Ospitalea. \\ Calle de Irunlarrea, 3. 31008 Pamplona.
}

Fecha de Recepción: 30/08/2021 - Fecha de Aceptación: 15/09/2021

En este trabajo se presenta la caracterización de la respuesta espectral de distintos modelos de paneles planos de yoduro de cesio. Se analizan 12 Flashpad de General Electric, 6 DRX Plus 4343C de Carestream, 4 CXDI-410 Wireless de Canon, 4 FDR D-EVO II de Fujifilm Corporation y 2 Pixium EZ de Trixell. Esta caracterización se Ileva a cabo midiendo la relación entre el Índice de Exposición y el kerma en aire incidente para haces con distinto espectro y se aportan resultados para cada modelo. Se propone también un método para la verificación del ajuste del menú anatómico empleando esta caracterización.

Palabras clave: Índice de Exposición, Radiología Digital, optimización, respuesta espectral.

This work provides the characterization of the spectral response of caesium iodide flat panel detectors from several manufacturers. The analysis is done for the following model flat panels: 12 Flashpad from General Electric, 6 DRX Plus 4343C from Carestream, 4 CXDI-410 Wireless from Canon, 4 FDR D-EVO II from Fujifilm Corporation and 2 Pixium EZ from Trixell. The characterization is carried out by measuring Exposure Index - incident air kerma ratios for beams with different spectrum and results are provided for each flat panel model. Furthermore, it is proposed a methodology to verify the correct configuration of the anatomic menu.

Key words: Exposure Index, Digital Radiography, optimization, spectral response.

\section{Introducción}

En los sistemas de radiología digital es de gran utilidad conocer la cantidad de radiación que se ha empleado para la formación de cada imagen. Esto permite distinguir tanto las subexposiciones como las sobreexposiciones o comparar el ajuste de distintos sistemas de imagen para una misma proyección. Con el fin de ofrecer esta información, cada fabricante desarrolló su propio indicador de la dosis que llegaba al receptor de imagen. Sin embargo, había una amplia variedad en la dependencia funcional con respecto al kerma en aire incidente $K_{a, i}$ y las condiciones de exposición de referencia eran diferentes para cada indicador (véase tabla 1) según se desprende del informe del Task Group 116 de la AAPM. ${ }^{1}$

En el año 2008 la Comisión Electrotécnica Internacional (IEC), publicó el documento IEC 62494$1: 2008,{ }^{2}$ que buscaba estandarizar y unificar los indicadores de dosis a través del Índice de Exposición (EI). Tal y como se indica en este documento, el Índice de Exposición debe calcularse según la expresión:

$$
E I=100 \mu G y^{-1} \cdot f^{-1}(V)
$$

\footnotetext{
*Correspondencia: aitor.fernandez.iglesias@navarra.es

** Trabajo presentado en el $3^{\text {er }}$ Congreso Europeo de Física Médica (ECMP) (16-19 Junio, Turín, Italia) y becado por la SEFM para la asistencia al mismo.

https://doi.org/10.37004/sefm/2021.22.2.005
} 
Tabla 1. Algunos ejemplos de indicadores de dosis y sus condiciones de calibración. Fuente: Adaptación de tabla C1 del Informe del Task Group 116 de la AAPM. ${ }^{1}$

\begin{tabular}{c|c|c|c|c} 
Fabricante & Indicador & $\begin{array}{c}\text { Dependencia con } \\
\text { exposición }\end{array}$ & kV/filtro estándar & Condiciones de calibración \\
\hline Agfa & $\operatorname{lgM}$ & Directa & $75 / 1.5 \mathrm{~mm} \mathrm{Cu}$ & 1.96 bel a $2.5 \mathrm{nGy}$ \\
\hline Kodak & EI & Directa & $80 / 0.5 \mathrm{~mm} \mathrm{Cu+1} \mathrm{mm} \mathrm{Al}$ & 2000 a $1 \mathrm{mR}$ \\
\hline Fujifilm & $\mathrm{S}$ & Inversa & $80 / 3 \mathrm{~mm} \mathrm{Al}$ (total) & 200 a $1 \mathrm{mR}$ \\
\hline GE & UDExp & Directa & $80 / 21 \mathrm{~mm} \mathrm{Al}$ & 2.85 a $0.5 \mathrm{mAs}$ \\
\hline
\end{tabular}

donde $V$ es una medida de tendencia central (como la media, la mediana o la moda) de los valores de píxel de la imagen for Processing que son clínicamente relevantes, y $f$ es la función de calibración del Índice de Exposición. Las condiciones de calibración que se definen en este documento son una tensión del tubo de rayos $\mathrm{X}$ comprendida entre 66 y $74 \mathrm{kV}$, un filtro añadido de $21 \mathrm{~mm}$ de aluminio y una capa hemirreductora $(\mathrm{CHR})$ de $6.8 \pm 0.3 \mathrm{~mm}$ de aluminio. Bajo estas condiciones de calibración, la función $f$ relaciona el kerma en aire incidente del haz primario con el valor de píxel producido cuando se irradia el panel homogéneamente, por lo que podemos sustituir la ecuación (1) por la siguiente:

$$
E I=\left.100 \cdot K_{a, i}(\mu G y)\right|_{\text {cond.cal. }}
$$

Sin embargo, esta expresión (2) es solamente válida bajo las condiciones de calibración, ya que la eficiencia de nuestro detector varía si la calidad del haz incidente cambia. Dicho de otro modo, un mismo valor de kerma en aire incidente proveniente de haces con diferente espectro produce valores de píxel distintos y, por consiguiente, índices de exposición distintos. Por lo tanto, de forma general podemos decir que se cumple lo siguiente:

$$
E I=C \cdot K_{a, i}(\mu G y)
$$

donde $C$ es un factor que depende de la calidad del haz de radiación. Para poder estimar el kerma en aire incidente en el receptor de imagen a partir del Índice de Exposición es necesario caracterizar la respuesta espectral de éste.

\section{Materiales y métodos}

Este trabajo ha consistido en caracterizar la respuesta espectral de varios paneles planos de yoduro de cesio (Csl) de distintas casas comerciales: 12 paneles planos modelo Flashpad de General Electric, 6 paneles planos modelo DRX Plus 4343C de Carestream, 4 paneles planos modelo CXDI-410 Wireless de Canon, 4 paneles planos modelo FDR D-EVO II de Fujifilm Corporation y 2 paneles planos modelo Pixium EZ de Trixell.

La caracterización la hemos llevado a cabo midiendo ratios $E I / K_{a, i}$, es decir, hemos medido los coeficientes $C$ de la ecuación (3) para distintas calidades de haz. Para ello, se han seleccionado diferentes combinaciones de tensión del tubo de rayos $X$ y espesores de atenuación. La tensión del tubo ha variado desde 40 kV hasta $130 \mathrm{kV}$ y el atenuador empleado ha sido metacrilato (PMMA) con espesores nominales entre 5 y 25 $\mathrm{cm}$. En todas las medidas el atenuador se ha situado a la salida del haz y el detector se ha colocado a una distancia foco-detector como mínimo de $150 \mathrm{~cm}$, con el fin de evitar la contribución de la radiación dispersa hacia adelante. Para las medidas de $K_{a, i}$ se ha empleado un multímetro Nomex de PTW, que presenta unas características válidas para el rango de calidades de haz que se ha empleado. Estas medidas de $K_{a, i}$ se han llevado a cabo colimando el campo a un tamaño de aproximadamente el tamaño de campo de calibración del medidor y la carga de cada disparo se ha escogido de forma que el $K_{a, i}$ medido esté en torno a 2 - $3 \mu \mathrm{Gy}$. A continuación, para medir el El, se ha colocado el panel plano en el lugar del Nomex y se ha realizado un disparo con la misma técnica de adquisición, pero abriendo el campo de forma que cubra todo el panel (ver figura 1). Si no es posible colocar el panel plano a la misma distancia que el Nomex, se debe corregir la lectura por inverso del cuadrado de la distancia. Finalmente, se han obtenido las ratios $E I / K_{a, i}$ dividiendo el valor del El por el correspondiente $K_{a, i}$ medido.

No obstante, el Índice de Exposición puede no estar ajustado correctamente, de forma que la ecuación (2) no se satisfaga. En estos casos, las casas comerciales suelen dar la opción de realizar una calibración siguiendo un procedimiento que a veces no es trivial. Esto supone que las ratios $E I / K_{a, i}$ son susceptibles de variar en el tiempo dependiendo de protocolos de ajuste del $E I$, espesores de atenuación empleados, empleo de medidores etc. Por ello, se pueden encontrar discrepancias entre valores de $E I / K_{a, i}$ para paneles del mismo 


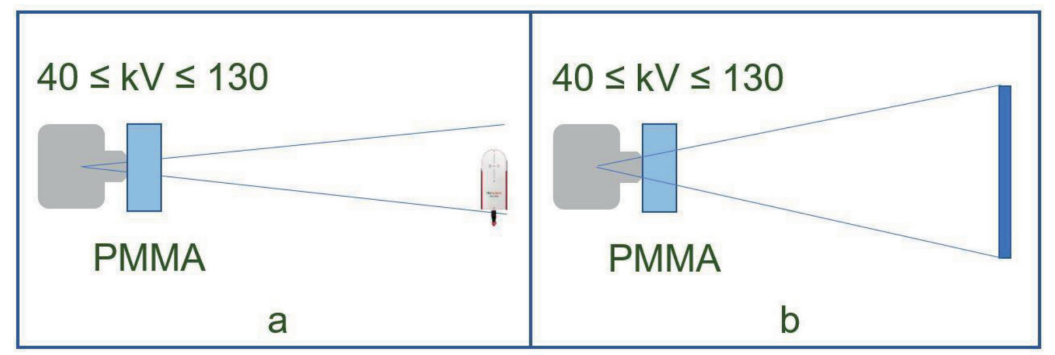

Fig. 1. Montaje experimental para la obtención de las ratios $E I / K_{a, i}$ : a) Condiciones para medir el $K_{a, i}$. b) Condiciones para medir el El. Fuente: Elaboración propia.

modelo, e incluso para un mismo panel dependiendo de cómo se ha realizado el último ajuste del El. Con el fin de evitar esta dependencia, la caracterización de la respuesta espectral de los distintos paneles planos se ha llevado a cabo dividiendo todas las ratios $E I / K_{a, i}$ por el valor de $E I / K_{a, i}$ que hemos medido en unas condiciones de referencia, obteniendo así unos factores relativos:

$$
f_{r e l}(k V, P M M A)=\frac{E I / K_{a, i}(k V, P M M A)}{E I / K_{a, i}\left(k V_{r e f}, P M M A_{r e f}\right)}
$$

Las condiciones de referencia que se han elegido son una tensión del tubo de rayos $\mathrm{X}$ de $80 \mathrm{kV}$ y un espesor de $15 \mathrm{~cm}$ de PMMA por ser ésta representativa de una técnica central.

Para poder obtener los factores relativos en unas condiciones de exposición que difieran de las combinaciones medidas, se ha parametrizado la dependencia del factor con respecto al kV y al espesor de PMMA. Concretamente, se ha realizado un ajuste por mínimos cuadrados a un polinomio de grado 3 con la siguiente forma:

$$
\begin{aligned}
f_{r e l}(x, y) & =a_{0}+a_{x} \cdot x+a_{y} \cdot y+a_{x^{2}} x^{2} \\
& +a_{y^{2}} \cdot y^{2}+a_{x y} \cdot x \cdot y \\
& +a_{x^{3}} \cdot x^{3}+a_{y^{3}} \cdot y^{3}+a_{x^{2} y} \cdot x^{2} \cdot y \\
& +a_{x y^{2}} \cdot x \cdot y^{2}
\end{aligned}
$$

donde la variable $x$ representa la tensión en kV y la variable $y$ representa el espesor de PMMA en $\mathrm{cm}$. Este ajuste se ha realizado para cada panel plano, para cada conjunto de paneles planos del mismo modelo y tam- bién uno común para todos los paneles analizados en este trabajo.

Por otro lado, se ha realizado un cálculo teórico de los factores relativos y se ha comparado con las medidas. Según John M. Boone ${ }^{3}$ la señal que genera un panel plano está relacionada con la energía total absorbida en el detector y la energía absorbida puede calcularse como sigue:

$$
E_{a b s}=\int_{E=0}^{E_{\max }} \Phi(E) E\left(\frac{\mu_{e n}(E)}{\mu(E)}\right)\left(1-e^{-\frac{\mu(E)}{\rho} \rho x}\right) d E
$$

donde: $\rho x$ es el espesor másico del cristal detector, $\frac{\mu(E)}{\rho}$ es el coeficiente de atenuación lineal másico del $C_{s}^{\rho} I$ para un haz de energía $E, \mu_{e n}(E)$ es el coeficiente de absorción del $C s I$ para un haz de energía $E$ y $\Phi(E)$ es la fluencia de fotones por intervalo de energía. A través de un simulador de espectros * se han generado algunos de los espectros correspondientes a las combinaciones kV - espesor de PMMA que hemos medido experimentalmente. Para ello, se ha supuesto una ventana de 2 $\mathrm{mm}$ de berilio y se ha añadido aluminio hasta que el haz resultante alcanzara una capa hemirreductora igual a la obtenida en las pruebas del control del tubo-generador. A continuación, se ha variado el kV y la filtración de PMMA manteniendo constante el kerma del haz resultante. Por otro lado, se han empleado los coeficientes de atenuación y absorción másica de la base de datos del NIST ${ }^{* *}$ para el CsI y se ha aplicado la ecuación (6) de forma discreta para obtener los factores relativos:

$$
f_{r e l}(k V, P M M A)=\frac{\sum_{i} \Phi\left(E_{i} ; k V, P M M A\right) \cdot \Delta E_{i} \cdot E_{i} \cdot \frac{\mu_{e n}\left(E_{i}\right)}{\mu\left(E_{i}\right)} \cdot\left(1-e^{-\frac{\mu\left(E_{i}\right)}{\rho} \rho d}\right)}{\sum_{i} \Phi\left(E_{i} ; 80,15 \mathrm{~cm}\right) \cdot \Delta E_{i} \cdot E_{i} \cdot \frac{\mu_{e n}\left(E_{i}\right)}{\mu\left(E_{i}\right)} \cdot\left(1-e^{-\frac{\mu\left(E_{i}\right)}{\rho} \rho d}\right)}
$$

* Siemens Healthineers, «Simulation of X-ray Spectra» Siemens Healthcare, [En línea]. Disponible: https://www.oem-products. siemens-healthineers.com/X-ray-spectra-simulation

** Hubbell, J.H. y Seltzer, S.M. (2004), Tables of X-Ray Mass Attenuation Coefficients and Mass Energy-Absorption Coefficients (version 1.4). [En línea] Disponible: http://physics.nist.gov/xaamdi [11/08/2021]. National Institute of Standards and Technology, Gaithersburg, MD. 
Tabla 2. Coeficientes de ajuste para cada modelo de panel plano analizado y coeficientes del ajuste común para todos los paneles planos analizados. Tal y como se indica en la expresión (6) todos los coeficientes tienen las unidades correspondientes para que al introducir la tensión del tubo en $\mathrm{kV}$ y el espesor de PMMA en $\mathrm{cm}$ el $f_{\text {rel }}$ sea adimensional. Fuente: Elaboración propia.

\begin{tabular}{c|c|c|c|c|c|c} 
& TRIXELL & CANON & CARESTREAM & FUJI & GE & COMÚN \\
\hline$a_{0}$ & $-6.808 \mathrm{E}-01$ & $-1.148 \mathrm{E}+00$ & $-9.204 \mathrm{E}-01$ & $-1.026 \mathrm{E}+00$ & $-6.404 \mathrm{E}-01$ & $-8.831 \mathrm{E}-01$ \\
\hline$a_{x}$ & $2.642 \mathrm{E}-02$ & $4.801 \mathrm{E}-02$ & $3.639 \mathrm{E}-02$ & $3.204 \mathrm{E}-02$ & $2.514 \mathrm{E}-02$ & $3.360 \mathrm{E}-02$ \\
\hline$a_{y}$ & $1.231 \mathrm{E}-02$ & $1.846 \mathrm{E}-02$ & $1.136 \mathrm{E}-02$ & $3.951 \mathrm{E}-02$ & $8.947 \mathrm{E}-03$ & $1.812 \mathrm{E}-02$ \\
\hline$a_{x^{2}}$ & $-1.210 \mathrm{E}-04$ & $-3.910 \mathrm{E}-04$ & $-2.470 \mathrm{E}-04$ & $-1.619 \mathrm{E}-04$ & $-1.134 \mathrm{E}-04$ & $-2.069 \mathrm{E}-04$ \\
\hline$a_{y^{2}}$ & $-8.516 \mathrm{E}-04$ & $-8.280 \mathrm{E}-04$ & $-1.269 \mathrm{E}-03$ & $-2.408 \mathrm{E}-03$ & $-9.008 \mathrm{E}-04$ & $-1.251 \mathrm{E}-03$ \\
\hline$a_{x y}$ & $4.023 \mathrm{E}-04$ & $2.720 \mathrm{E}-04$ & $5.455 \mathrm{E}-04$ & $3.216 \mathrm{E}-04$ & $5.318 \mathrm{E}-04$ & $4.146 \mathrm{E}-04$ \\
\hline$a_{x^{3}}$ & $6.325 \mathrm{E}-08$ & $1.089 \mathrm{E}-06$ & $5.837 \mathrm{E}-07$ & $2.037 \mathrm{E}-07$ & $7.603 \mathrm{E}-08$ & $4.031 \mathrm{E}-07$ \\
\hline$a_{y^{3}}$ & $1.660 \mathrm{E}-05$ & $6.421 \mathrm{E}-06$ & $1.272 \mathrm{E}-05$ & $3.882 \mathrm{E}-05$ & $9.519 \mathrm{E}-06$ & $1.682 \mathrm{E}-05$ \\
\hline$a_{x^{2} y}$ & $-1.991 \mathrm{E}-06$ & $-2.140 \mathrm{E}-06$ & $-2.770 \mathrm{E}-06$ & $-2.373 \mathrm{E}-06$ & $-2.890 \mathrm{E}-06$ & $-2.433 \mathrm{E}-06$ \\
\hline$a_{x y^{2}}$ & $-3.110 \mathrm{E}-06$ & $1.074 \mathrm{E}-06$ & $-1.390 \mathrm{E}-06$ & $1.131 \mathrm{E}-06$ & $-1.892 \mathrm{E}-06$ & $-8.374 \mathrm{E}-07$
\end{tabular}

\section{Resultados}

En la tabla 2 se muestran los coeficientes del ajuste (6) obtenidos para cada modelo de panel plano estudiado junto con los coeficientes del ajuste común. La desviación entre el factor relativo que se obtiene a partir del ajuste y el factor relativo medido experimentalmente se encuentra por debajo del 8\% para todas las combinaciones. Más allá de esta diferencia se encuentran combinaciones de PMMA - kV que por tener un $\mathrm{kV}$ o un espesor extremo se alejan de las condiciones clínicas.
En la tabla 3 se muestran como ejemplo los valores de $E I / K_{a, i}$ medidos para uno de los paneles planos modelo Flashpad de General Electric. Se muestra también la desviación entre los factores relativos medidos y los obtenidos mediante los coeficientes de ajuste de la tabla 2 correspondientes al modelo Flashpad.

Por otro lado, en la tabla 4 se muestran los factores relativos teóricos estimados a partir de la expresión (7) para un espesor del cristal de $200 \mu \mathrm{m}$. En esta misma tabla se muestra también la comparación de los factores relativos teóricos con los factores relativos que obtenemos para cada modelo a partir de los coeficientes de ajuste de la tabla 2. Puesto que no se conoce el espesor

Tabla 3. Valores de $E I / K_{a, i}$ medidos para un panel plano modelo Flashpad de General Electric. Comparación con los factores relativos calculados a través de los coeficientes de ajuste del modelo que aparecen en la tabla 2. Fuente: Elaboración propia.

\begin{tabular}{c|c|c|c|c|c}
$\mathrm{kV}$ & Espesor $(\mathrm{cm})$ & $E I / K_{a, i}$ & $f_{\text {rel }}$ medido & $f_{\text {rel }}$ ajustado & Desv. \\
\hline 60 & 10 & 61.943 & 0.705 & 0.688 & $-2.40 \%$ \\
\hline 80 & 10 & 86.112 & 0.981 & 0.918 & $-6.42 \%$ \\
\hline 80 & 15 & 87.822 & 1.000 & 0.974 & $-2.63 \%$ \\
\hline 80 & 20 & 91.785 & 1.045 & 0.999 & $-4.45 \%$ \\
\hline 100 & 10 & 93.334 & 1.063 & 1.048 & $-1.42 \%$ \\
\hline 100 & 15 & 98.845 & 1.126 & 1.100 & $-2.26 \%$ \\
\hline 120 & 10 & 96.664 & 1.101 & 1.082 & $-1.70 \%$ \\
\hline 120 & 15 & 102.021 & 1.162 & 1.119 & $-3.64 \%$
\end{tabular}


Tabla 4. Comparación de los factores relativos obtenidos mediante la expresión (7) y los medidos experimentalmente. Fuente: Elaboración propia.

\begin{tabular}{c|c|c|c|c|c|c|c}
$\mathrm{kV}$ & Esp. PMMA (cm) & $f_{\text {rel }}(\mathrm{CsI} \mathbf{2 0 0} \mu \mathrm{m})$ & TRIXELL & CANON & CARESTREAM & FUII & GE \\
\hline 60 & 10 & 0.726 & $5.58 \%$ & $-4.55 \%$ & $1.10 \%$ & $9.24 \%$ & $5.47 \%$ \\
\hline 70 & 15 & 0.916 & $4.89 \%$ & $-1.24 \%$ & $2.74 \%$ & $7.01 \%$ & $5.37 \%$ \\
\hline 80 & 15 & 1.000 & $2.51 \%$ & $-0.05 \%$ & $-0.03 \%$ & $3.55 \%$ & $2.64 \%$ \\
\hline 80 & 20 & 1.056 & $3.64 \%$ & $2.18 \%$ & $5.13 \%$ & $6.63 \%$ & $5.64 \%$ \\
\hline 80 & 25 & 1.099 & $4.04 \%$ & $4.90 \%$ & $12.50 \%$ & $8.30 \%$ & $9.89 \%$ \\
\hline 100 & 10 & 0.998 & $-4.09 \%$ & $-1.04 \%$ & $-8.09 \%$ & $-5.04 \%$ & $-4.76 \%$ \\
\hline 100 & 20 & 1.090 & $-3.99 \%$ & $0.85 \%$ & $-4.01 \%$ & $-2.20 \%$ & $-2.75 \%$ \\
\hline 120 & 15 & 1.023 & $-8.35 \%$ & $-1.03 \%$ & $-12.76 \%$ & $-8.55 \%$ & $-8.73 \%$ \\
\hline 120 & 20 & 1.036 & $-8.91 \%$ & $-0.55 \%$ & $-10.99 \%$ & $-7.45 \%$ & $-7.70 \%$ \\
\hline
\end{tabular}

del cristal de CsI de los distintos modelos se ha elegido el espesor que hace que la diferencia entre los factores relativos teóricos y medidos sea mínima.

\section{Discusión}

Los resultados de la tabla 3 muestran que la eficiencia del detector varía considerablemente en función de la calidad del haz incidente. En general, se observa que la eficiencia de los paneles analizados es inferior para tensiones del tubo de rayos $X$ bajos y espesores de atenuación bajos. Estas condiciones pueden encontrarse tanto en exploraciones de extremidades como en exploraciones pediátricas. Por lo que la aplicación de la expresión (2) para estimar el $K_{a, i}$ a partir del $E I$ en estos casos, puede suponer cometer un error por encima del $30 \%$.

Por otro lado, esta caracterización puede ser de gran utilidad para verificar el ajuste del menú anatómico en los sistemas digitales. Si se dispone de los factores relativos (4) que caracterizan la respuesta espectral del panel, los pasos para la estimación del $K_{a, i}$ serían los siguientes. En primer lugar, para evitar que un desajuste del índice de exposición influya en el resultado final, debemos medir el valor de $E I / K_{a, i}\left(k V_{r e f}, P M M A_{r e f}\right)$ en las condiciones de referencia. Para ello, debe realizarse una adquisición con la tensión del tubo $k V_{\text {ref }} \mathrm{y}$ el espesor de atenuación $P M M A_{\text {ref }}$ correspondientes e irradiar el panel completo de forma homogénea. Es recomendable colocar el PMMA a la salida del haz para asegurar que el panel queda completamente cubierto y obtener el $E I$ con el panel en su alojamiento, ya que algunos fabricantes emplean una calibración del EI independiente para las adquisiciones que se realizan con el panel libre. En este caso, el $K_{a, i}$ debe estimarse bien sea mediante el valor medio de píxel de la imagen For processing y aplicando la función de calibración $f$ o midiéndolo con un detector y aplicando factores de atenuación si fuera necesario. Una vez se ha determinado el $K_{a, i}$, se divide el El por el $K_{a, i}$ y se obtiene el valor de $\left.E I / K_{a, i}\left(k V_{\text {ref }}, P M M A_{r e f}\right)\right)$. A continuación, se coloca el espesor de PMMA que sea representativo del espesor del paciente para la proyección en la que se quiere determinar el kerma en aire incidente y se adquiere con la técnica correspondiente. El siguiente paso consistiría en introducir el espesor de PMMA y el kV empleado para la adquisición en la ecuación (5) obteniendo el factor relativo. Después, se aplica la expresión (4) para obtener el valor de $E I / K_{a, i}(k V, P M M A)$. Finalmente, el $K_{a, i}$ se obtiene dividiendo el valor del El de la proyección por el valor de $E I / K_{a, i}(k V, P M M A)$ calculado.

Este método permite comprobar que el $K_{a, i}$ con el que corta el control automático de exposición (CAE) para una proyección dada no ha variado de un control de calidad a otro y también permite comparar el $K_{a, i}$ que emplean los distintos sistemas para una misma proyección. No obstante, es necesario vincular el $K_{a, i}$ con parámetros como la relación señal-ruido (RSR) o figuras de índice de calidad (IQF), para determinar cuál es el $K_{a, i}$ óptimo para cada tarea.

\section{Conclusiones}

En este trabajo se presenta la caracterización espectral de varios modelos de paneles planos que se encuentran actualmente en el mercado. Siguiendo el método propuesto puede estimarse de forma sencilla 
el $K_{a, i}$, aunque es necesario trabajar con parámetros como la RSR o IQF para establecer el $K_{a, i}$ óptimo para cada tarea.

\section{Referencias}

1. American Association of Physicists in Medicine. An Exposure Indicator for Digital Radiography, Report of AAPM Task Group 116. College Park, MD: AAPM; 2009.
2. International Electrotechnical Commission (IEC), Medical Electrical Equipment: Exposure Index of Digital X-Ray Imaging Systems. Definitions and requirements for general radiography, Geneva, Switzerland, 2008.

3. Boone J.M., X-ray production, interaction and detection in diagnostic imaging. In: Beutel J., Kundel H.L., Van Metter R.L. (eds), Handbook of medical imaging, Vol1, Physics and Psychophysics., SPIE, Bellingham, 2000. 


\section{imagine}

